\title{
An orthogonal, one-pot, simultaneous UV-mediated route to thiol-ene/sol-gel film
}

\author{
A. Chemtob ${ }^{1}$, H. De Paz-Simon ${ }^{1}$, M. Sibeaud ${ }^{1}$, B. El Fouhali ${ }^{1}$, C. Croutxé-Barghorn ${ }^{1}$, \\ L. Jacomine ${ }^{2}$, Ch. Gauthier ${ }^{2}$, V. Le Houérou ${ }^{2}$ \\ ${ }^{1}$ Laboratory of Macromolecular Photochemistry and Engineering, University of Haute-Alsace, EA 4567, ENSCMu, \\ 3 bis rue Alfred Werner, 68093 Mulhouse Cedex, France \\ ${ }^{2}$ Institut Charles Sadron, CNRS, UPR 22, University of Strasbourg, 23 Rue du Loess, BP 84047, 67034 Strasbourg \\ Cedex 2, France
}

Received 9 October 2015; accepted in revised form 22 December 2015

\begin{abstract}
We describe a novel combination of orthogonal reactions based on UV-driven thiol-ene and alkoxysilyl condensation reactions to form a single-step route toward thioether-bridged silsesquioxane films. Our chemical strategy consists of using two bifunctional (meth)acrylate (E) and propanethiol (T) trimethoxysilyl precursors containing two complementary functional moieties for thiol-ene coupling and sol-gel process. The reaction kinetics revealed that c.a. $85 \%$ of thiol and ene conversions were consumed concomitantly. Meanwhile, a complete hydrolysis was accomplished, affording ultimately a high degree of condensation (81\%). Emphasis was placed on differences of mechanical properties between sol-gel hybrids resulting from thiol-ene reaction (E-T mixture) and ene homopolymerization (E only) using scratch test measurements. For the methacrylate system, the formation of thioether linkages within a vitreous silica network emerged as a useful strategy for the formation of a uniform, low-stress and flexible crosslinked hybrid structure. Enhanced mechanical properties were manifested by an expanded elastic domain, and better resistance to cracking. Moreover, there are clear indications that mechanical properties can be easily tuned upon varying the ratio of the two hybrid precursors.
\end{abstract}

Keywords: mechanical properties, thiol-ene, nanocomposites, photopolymerization, UV irradiation

\section{Introduction}

The revival of 'thiol-ene' reaction, which refers to the radical-mediated addition of thiol across terminal alkene, hinges mainly on some essential factors: accessibility of raw materials, mild reaction conditions, efficiency and chemoselectivity $[1,2]$. The last feature refers to the fact that this coupling reaction can be 'orthogonal' to a broad array of reagents, solvents, and functional groups. Thiol-ene orthogonality [3] has already paved the way for step-growth polymerizations and chain functionalizations performed in a wide range of chemical environments: aerated [4] and aqueous [5] conditions for example, or in the presence of biological [6] and complex [7] systems. The recent combination of thiol-ene with sol-gel chemistry provides another perfect illustration of this tolerance, because this inorganic polymerization process generally entails water and alcoholic solvents/byproducts, acid or base catalysts, and precursors bearing chloro or alkoxy hydrolyzable reactive groups [8]. However, most of the reported synthesis methodologies remain sequential describing a thiol-ene step uncoupled from the sol-gel process [9]. In most instances, the (photoinduced) thiol-ene cross-linking was thus carried out subsequently from isolated and purified thiol- or vinyl-functionalized hybrid building blocks including silsesquioxanes [10-17], POSS (polyoligomeric silsesquiox-

\footnotetext{
${ }^{*}$ Corresponding author, e-mail: abraham.chemtob@uha.fr

(C) BME-PT
} 
ane) cages $[18,19]$ or zirconium oxoclusters [20], all prepared separately. These approaches were derived from the conventional two-step route used to synthesize hybrid sol-gel photopolymer films via radical or cationic photopolymerization [21]. Complementing a thiol-ene network with an intimately mixed silica or oxide matrix is clearly part of a wider strategy to improve its mechanical properties, which are usually plagued by low glass transition temperature and poor cross-link densities [22]. Although the aforementioned approaches claimed notable improvements in properties such as abrasion resistance, thermal stability [12] or refractive index [23], they do not allow optimum use of thiol-ene orthogonality, raising hopes that synthesis conditions could be dramatically improved.
We describe herein a combination of two photoinduced orthogonal reactions based on thiol-ene and alkoxysilyl sol-gel condensation toward the rapid formation of thioether-siloxane nanocomposite films. As outlined in Figure 1, this novel approach focuses on simultaneous, one-pot reactions, thereby minimizing the number of required synthetic steps and reducing the number of work-up and purification operations. Our chemical strategy consists of using a bifunctional monomer - 3-(trimethoxysilyl)propyl methacrylate $\left(\mathbf{E}_{1}\right)$ or acrylate $\left(\mathbf{E}_{2}\right)$ - containing two complementary ene and trimethoxysilyl functional moieties for thiol-ene and sol-gel process, respectively. This latter is coupled with 3-(trimethoxysilyl)-1-propanethiol (T) to form a single-step route toward thioether-bridged silsesquioxane films (if no

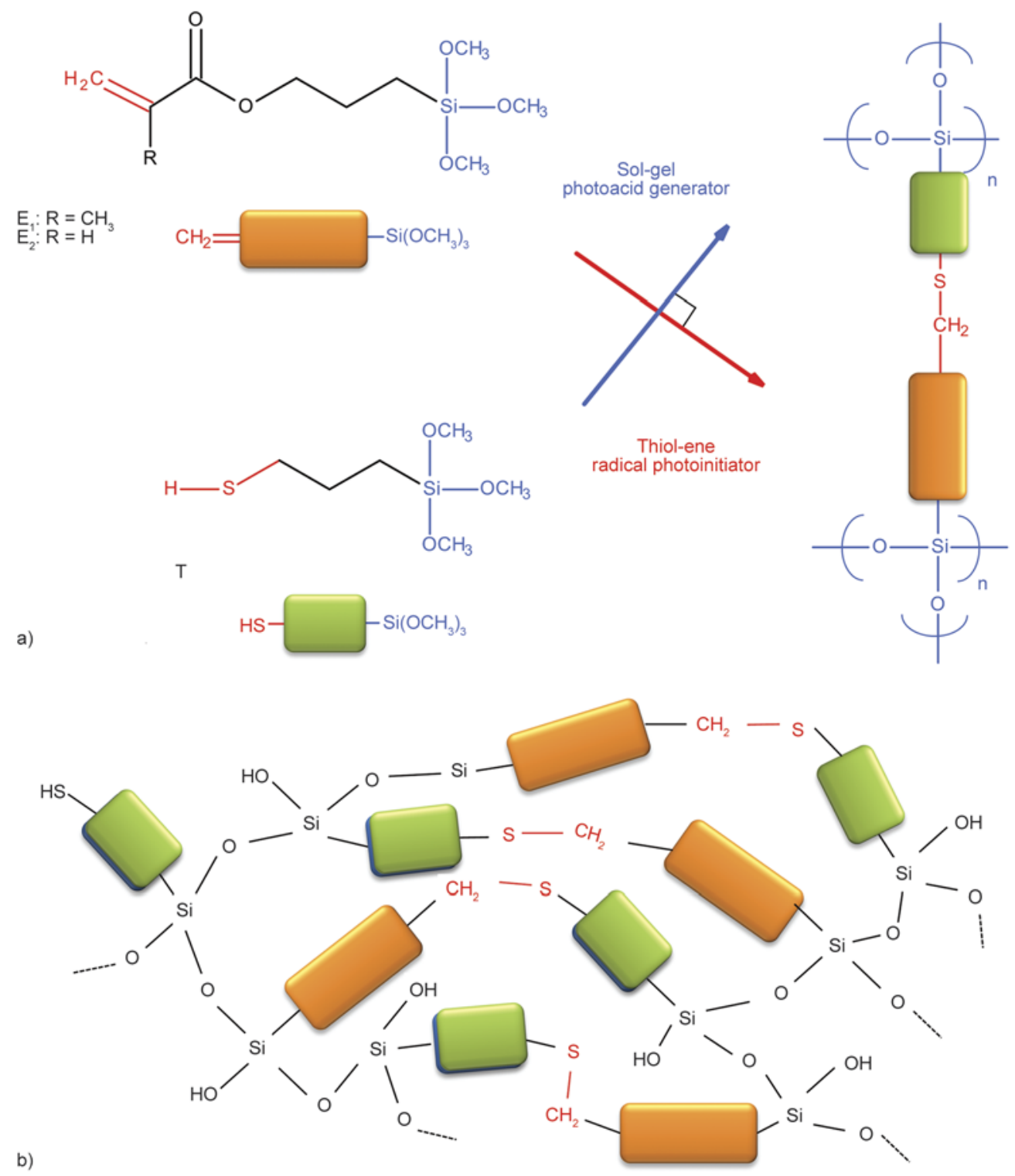

Figure 1. a) An orthogonal coupling strategy was developed by combining thiol-ene and sol-gel reactions for the synthesis of hybrid thiol-ene films. b) schematic representation of the thioether-bridged silsesquioxane network 
homopolymerization of ene occurs) represented in Figure 1. Thiol-ene is photochemically triggered by the aid of a radical photoinitiator, while sol-gel process is catalyzed through the release of photoacids. The concept of sol-gel photopolymerization of silicon alkoxide-based films has the peculiar advantages of proceeding in bulk, thus avoiding solvent or water addition, and sol-gel transition. Nevertheless, it proceeds through a hydrolysis-condensation mechanism, entirely similar to that of a conventional hydrolytic sol-gel process [24, 25]. Catalysis is mediated by photogenerated Brönsted superacids, while the hydrolysis stage relies on the permeation of air moisture. The high chemoselectivity of thiol-ene allows it to form a highly orthogonal reaction pair with photoacid-catalyzed hydrolysis-condensation of the trimethoxysilyl groups. Additionally, this route obviates the necessity for solvent, deprotection/ activation steps, or thermal densification of the oxopolymer network.

In contrast to chain-growth polymerization leading to multiple additions, thiol-ene is a true coupling reaction as a single $\mathrm{S}-\mathrm{C}$ bond arises from the net addition of thiol across an ene. The resulting benefit is the formation of a well-defined silsesquioxane network including fewer crosslinks and flexible thioether bridges able to offset silica brittleness and residual stress. Emphasis was placed on the possible differences of mechanical responses between sol-gel hybrids resulting from thiol-ene reaction and ene homopolymerization since little study has been done so far on this subject. In our case, the choice of (meth)acrylate precursors (E) able to undergo both reactions creates an opportunity to elucidate their distinct mechanical behaviors. Unlike main approaches in the literature based on dynamic mechanical analysis, we investigated surface properties through scratch test apparatus equipped with in situ visualization and image-analysis protocol. This technique can provide new insight into the adhesion of the hybrid films, elasto-plastic properties of the film material, fracture mechanism, and more generally resistance to scratch.

\section{Experimental section}

\subsection{Materials}

3-(Trimethoxysilyl)-1-propanethiol (T, $98 \mathrm{~mol} \%$ ), 3(trimethoxysilyl)propyl methacrylate $\left(\mathbf{E}_{\mathbf{1}}, 98 \mathrm{~mol} \%\right)$ and acrylate $\left(\mathbf{E}_{2}, 98 \mathrm{~mol} \%\right)$ were purchased from Sigma-Aldrich and used without further purifica- tion. 4-methylphenyl)[4-(2-methylpropyl)phenyl] iodonium hexafluorophosphate (Irgacure 250, $75 \mathrm{wt} \%$ in propylene carbonate) is a ionic photoacid generator provided by BASF and used to photocatalyze the inorganic photopolymerization. The radical photoinitiator for thiol-ene coupling or ene homopolymerization is 2-hydroxy-2-methyl-1-phenyl-propan1-one (Darocur 1173) kindly provided by BASF. The silicon wafer (100) substrate was purchased from SILTRONIX Silicon Technologies. Optically polished $\mathrm{BaF}_{2}$ IR windows $(25 \times 5 \mathrm{~mm})$ were purchased from Korth Kristalle GmbH.

\subsection{Hybrid thiol-ene film preparation}

$2 \mathrm{wt} \%$ of I 250 and D1173 were added to a T-E $\mathbf{E}_{\mathbf{i}}$ thiolene mixture containing an equimolar ratio in $\mathrm{SH}$ and $\mathrm{C}=\mathrm{C}$ functions. Before $\mathrm{UV}$ exposure, the homogeneous and solvent-free formulations were cast onto two different substrates $\left(\mathrm{BaF}_{2}\right.$ pellet or borosilicate glass) using a wire-wound bar to yield a liquid film with a thickness of $3.8 \pm 0.2 \mu \mathrm{m} . \mathrm{BaF}_{2}$ substrate was employed for real-time Fourier transform infrared (RT-FTIR) experiments. In this specific case, irradiation was performed at room temperature with simultaneous exposure to UV and IR analytical beam during $200 \mathrm{~s}$. As UV source, a polychromatic mercury-xenon lamp (Hamamatsu, L8252, $200 \mathrm{~W}$, $365 \mathrm{~nm}$ reflector) was used, which covers a broad continuous spectrum spanning from short-wavelength UV to infrared (250-2000 nm). This lamp was connected to a flexible light-guide to generate a spot light beam on the film sample and an elliptical cold reflector was added to prevent any adverse effects from heat (IR radiation). In the spectral region between 250 and $600 \mathrm{~nm}$, the light irradiance I was estimated at $600 \mathrm{~mW} \cdot \mathrm{cm}^{-2}$. After the irradiation process, the temperature increase was very limited $\left(<2{ }^{\circ} \mathrm{C}\right)$, neglecting any thermal contribution to the sol-gel process. All measurements were repeated at least three times and reproducible results were obtained. Larger films dedicated to mechanical characterization and solid-state NMR analysis were prepared with the same formulation deposited on glass substrate previously cleaned with acetone and ethanol. In this second case, irradiation was performed at room temperature under an industrial UV conveyor using a microwave $\mathrm{Hg}$ arc lamp (H lamp, Fusion). The belt speed of the conveyor was set at $10 \mathrm{~m} / \mathrm{min}$ and the lamp intensity at $100 \%$. Under these conditions, for each pass, the emitted light dose 
is $1.46 \mathrm{~J} / \mathrm{cm}^{2}$ (UVA [320-390 nm]: $0.45 \mathrm{~J} / \mathrm{cm}^{2}$, UVB [280-320 nm]: $0.42 \mathrm{~J} / \mathrm{cm}^{2}$, UVC [250-260 nm]: $0.09 \mathrm{~J} / \mathrm{cm}^{2}$ and UVV [395-445 $\mathrm{nm}$ ]: $0.5 \mathrm{~J} / \mathrm{cm}^{2}$ ). Only 5 passes under the UV conveyor were performed to afford solid crosslinked films. For ene chain-growth polymerization (homopolymerization), the same procedure was repeated without including the thiolbased organosilane $(\mathbf{T})$.

\subsection{Characterization}

Time-resolved infrared spectra obtained by RTFTIR spectroscopy were recorded with a Bruker Vertex 70. The resolution of the infrared spectra was $4 \mathrm{~cm}^{-1}$. Liquid film thickness prior to irradiation was determined by optical profilometry using an Altisurf 500 workstation (Altimet) equipped with a $350 \mu \mathrm{m}$ AltiProbe optical sensor. During the UV irradiation, the absorbance decrease of the $\mathrm{CH}_{3}$ symmetric stretching vibration band centred at $2848 \mathrm{~cm}^{-1}$ (distinctive from $\mathrm{CH}_{3}$ methanol vibration modes) was monitored to follow the methoxysilyl hydrolysis in PDMOS. All spectra were baseline corrected prior to integration with the software OPUS 6.5. To obtain quantitatively reliable ${ }^{29} \mathrm{Si}$ and ${ }^{13} \mathrm{C}$ solid state NMR spectra, single pulse magic angle spinning (SPEMAS) experiments were performed on a Bruker Avance II 400 spectrometer operating at 79.48 and 100.6 MHz respectively with a Bruker double channel $7 \mathrm{~mm}$ probe. Spectra were recorded using a pulse angle of $\pi / 4$, a recycling delay of 80 and $60 \mathrm{~s}$ respectively, a spinning frequency of 4 or $5 \mathrm{kHz}$ and highpower proton decoupling during the acquisition. ${ }^{29} \mathrm{Si}$ and ${ }^{13} \mathrm{C}$ chemical shifts are both relative to tetramethylsilane. Deconvolution of the spectrum was performed using Dmfit software [26]. The scratch resistance of the UV-cured coatings deposited onto glass plate was characterized at room temperature using the apparatus described in previous articles [27]. The tip was a diamond sphere of radius $96 \mu \mathrm{m}$, and the sliding speed was maintained constant $(30 \mu \mathrm{m} / \mathrm{s})$. First of all, one should note that the penetration of the spherical indenter is at least 40 times smaller than the film thickness. As a result, this latter can be considered as a semi-infinite solid. The tests were performed under a dry atmosphere (Relative Humidity $<4 \%$ ). Stepwise normal load ramps were performed in the range of 0.04 to $10 \mathrm{~N}$. At each force step, the tip moved over a distance of $1 \mathrm{~mm}$ to obtain a groove that can be analyzed in terms of steady state regime. The tangential load was recorded throughout the experiment, and pictures were taken with the help of an in situ microscope equipped with a CCD camera. Then, the size and shape of the contacts on the coatings were analyzed through the transparent sample and substrate.

\section{Results and discussion \\ 3.1. Synthesis and characterization of thiol-ene hybrid films}

Based on real-time FTIR data, Figure $2 \mathrm{a}$ is a conversion-time plot $\left(\mathrm{SH}, \mathrm{C}=\mathrm{C}\right.$ and $\left.\mathrm{OSi}\left(\mathrm{OCH}_{3}\right)_{3}\right)$ during UV irradiation in an air environment of a $\mathbf{E}_{\mathbf{1}}-\mathbf{T}$ film including a stoichiometric ratio of thiol-ene functional groups. The formulation contains also $2 \mathrm{wt} \%$ diaryl iodonium salt photoacid generator (I250) and hydroxyphenylketone radical photoinitiator (D1173), to build-up the two silsesquioxane networks and the thioether bridges, respectively. The acid-catalyzed hydrolysis of $\mathrm{OSi}\left(\mathrm{OCH}_{3}\right)_{3}$ into $\mathrm{SiOH}$, which precedes condensation, occurs at very fast rates achiev-
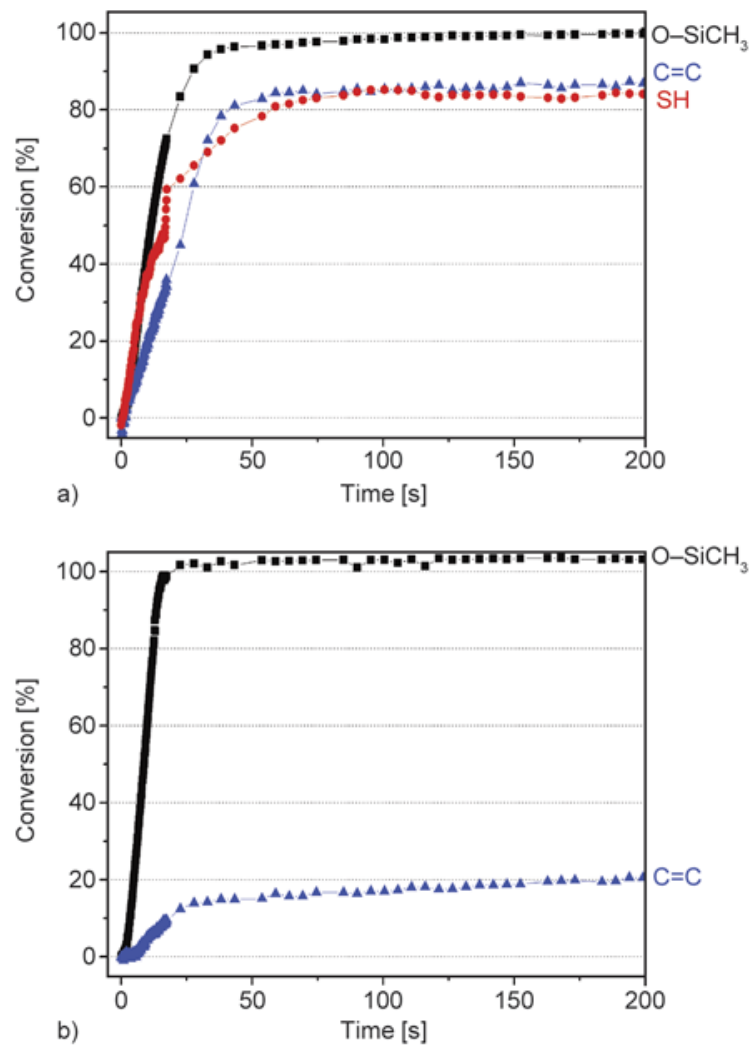

Figure 2. Temporal conversion in ene, thiol and methoxysilyl groups during the UV irradiation of $\mathbf{E}_{\mathbf{1}}$-T with a 1:1 thiol-ene stoichiometry (a) and $\mathbf{E}_{\mathbf{1}}$ (b). Kinetic data were obtained from integrated absorbance in the FTIR spectra: $\mathrm{C}=\mathrm{C}$ (triangle, $=\mathrm{CH}_{2}$ bending at $1310 \mathrm{~cm}^{-1}$ ), $\mathrm{SH}$ (circle, $\mathrm{S}-\mathrm{H}$ stretching at $2570 \mathrm{~cm}^{-1}$ ) $\mathrm{SiO}-\mathrm{CH}_{3}$ (square, $\mathrm{CH}_{3}$ asymmetric stretching at $\left.2848 \mathrm{~cm}^{-1}\right)$. 
ing completion after $20 \mathrm{~s}$ exposure. In addition, there are clear indications that thiol and methacrylate are consumed concomitantly, suggesting that a thiolene coupling is preferentially active compared to a conventional ene homopolymerization. Low film thickness $(\approx 4 \mu \mathrm{m})$ and viscosity $\left(2-3 \mathrm{mPa} \cdot \mathrm{s}\right.$ at $\left.25^{\circ} \mathrm{C}\right)$ are both conducive to oxygen permeation to form peroxy radicals. While these species are inactive in a chain-growth radical process, they can readily abstract a hydrogen atom from a thiol to form an active thiyl radical adding to an acrylate. Therefore, a single transfer/addition thiol-ene mechanism is likely to operate under these conditions, to yield a homogenous thioether-bridged silica network as sketched in Figure 1. Furthermore, because sol-gel photopolymerization is generally under a water per- meation controlled-regime [25], thin film conditions favour a fast a complete hydrolysis reaction. Under these conditions, thiol-ene reaction which takes place rapidly $(<25 \mathrm{~s})$, proceeds almost synchronously with hydrolysis. This latter feature is critical to ensure high conversion (85\%) as an early sol-gel vitrification is likely to impede the thiol-ene coupling reaction. Conversely, a conventional chain-growth polymerization can be favored by irradiating the single precursor $\mathbf{E}_{\mathbf{1}}$ film. In this second case, the silsesquioxane network architecture changes significantly (Figure 3 ). In conventional chain-growth polymerizations, each monomer is coupled to two other monomers units affording higher cross-link density in contrast to radical thiol-ene reaction in which each ene group forms only a single bond with<smiles>[R]C(=C)C(=O)OCCC[Si](O)(OC)OC</smiles>
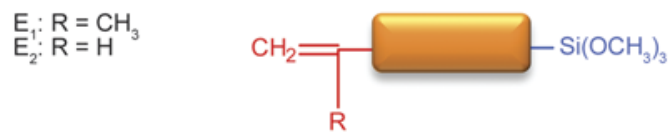

a)

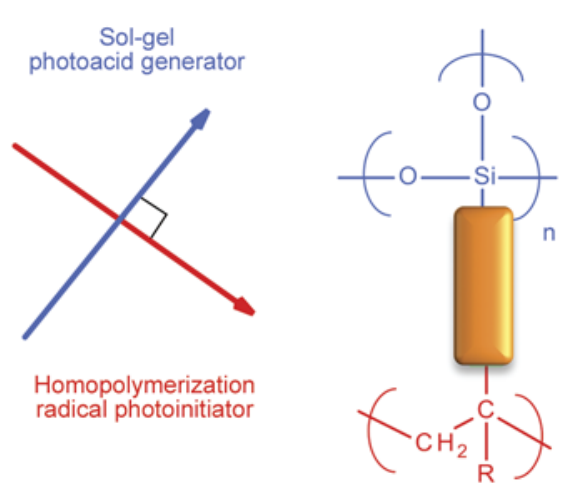

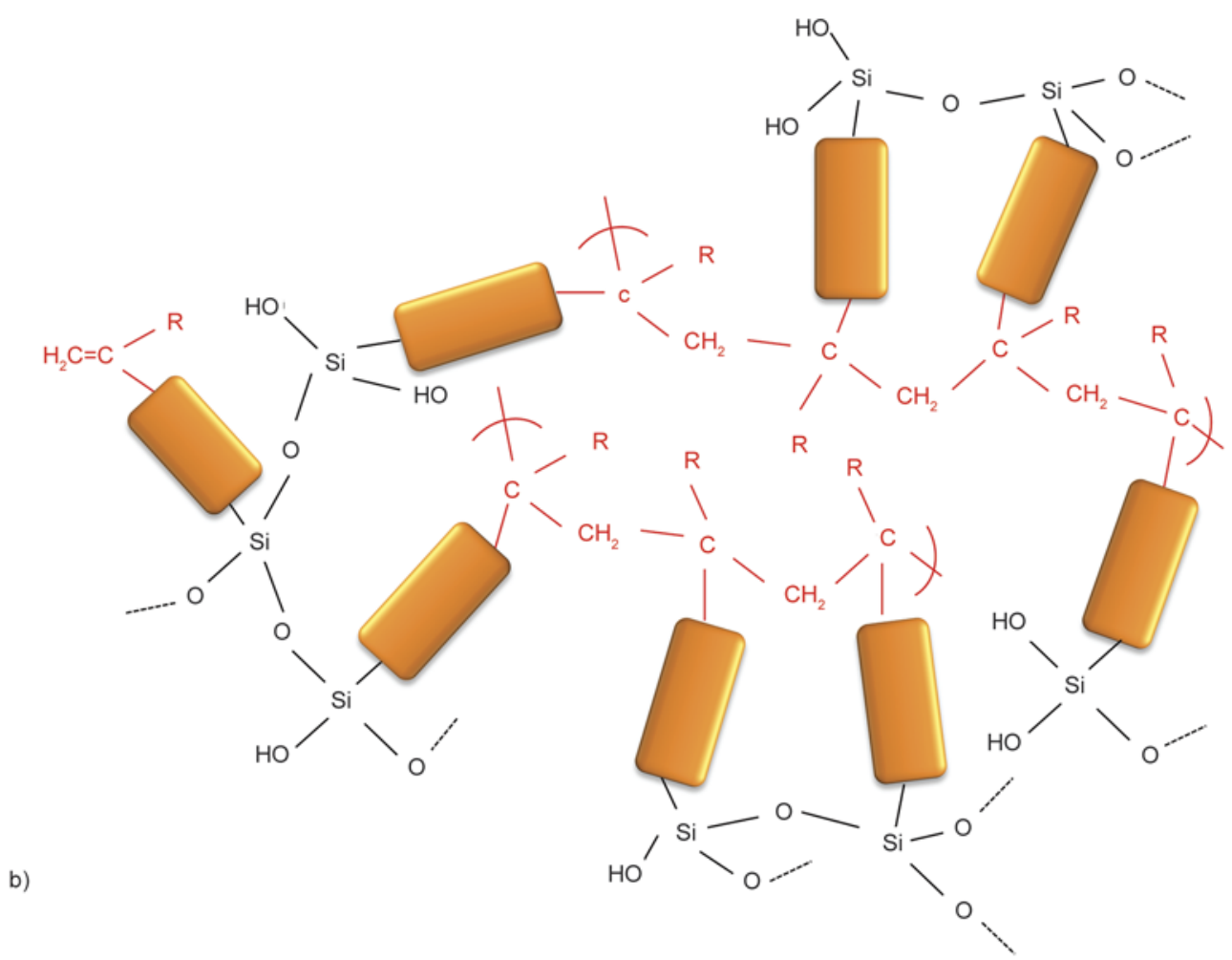

Figure 3. a) Dual ene homopolymerization and sol-gel process from precursors $\mathbf{E}_{\mathbf{1}}\left(\mathrm{R}=\mathrm{CH}_{3}\right)$ and $\mathbf{E}_{\mathbf{2}}(\mathrm{R}=\mathrm{H})$ affording a polymer-siloxane nanocomposite structure represented in b). 
another thiol. Figure $2 b$ displays the organic-inorganic kinetic curves for the nanocomposite film derived from $\mathbf{E}_{\mathbf{1}}$ irradiated alone. The hydrolysis rates remain unchanged with respect to the thiolene $\mathbf{E}_{\mathbf{1}}-\mathbf{T}$ mixture, whereas the consumption of the methacrylate moiety is significantly different. As expected, methacrylate homopolymerization is slower and strongly restricted by oxygen inhibition, which limits the conversion to ca. $20 \%$. However, the resultant polymethacrylate hybrid film is tack-free.

Likewise, a thiol-ene/sol-gel tandem reaction was carried out with the $\mathbf{E}_{\mathbf{2}}-\mathbf{T}$ pair. Figure 4 shows some kinetic profiles very similar to the first couple $(100 \%$ hydrolysis, $80 \%$ thiol-ene conversion). This suggests that this approach could accept a range of thiol- or ene-based silsesquioxane precursors. Compared with conventional routes to hybrid thiol-ene networks, a key feature of this tandem photosol-gel and photopolymerization is high reaction speed. Although the present data establish sol-gel and thiol-ene reactions as a robust orthogonal set of coupling reactions, the chemoselectivity must be mitigated by the fact that carboradicals from radical PI or thiyl radi-
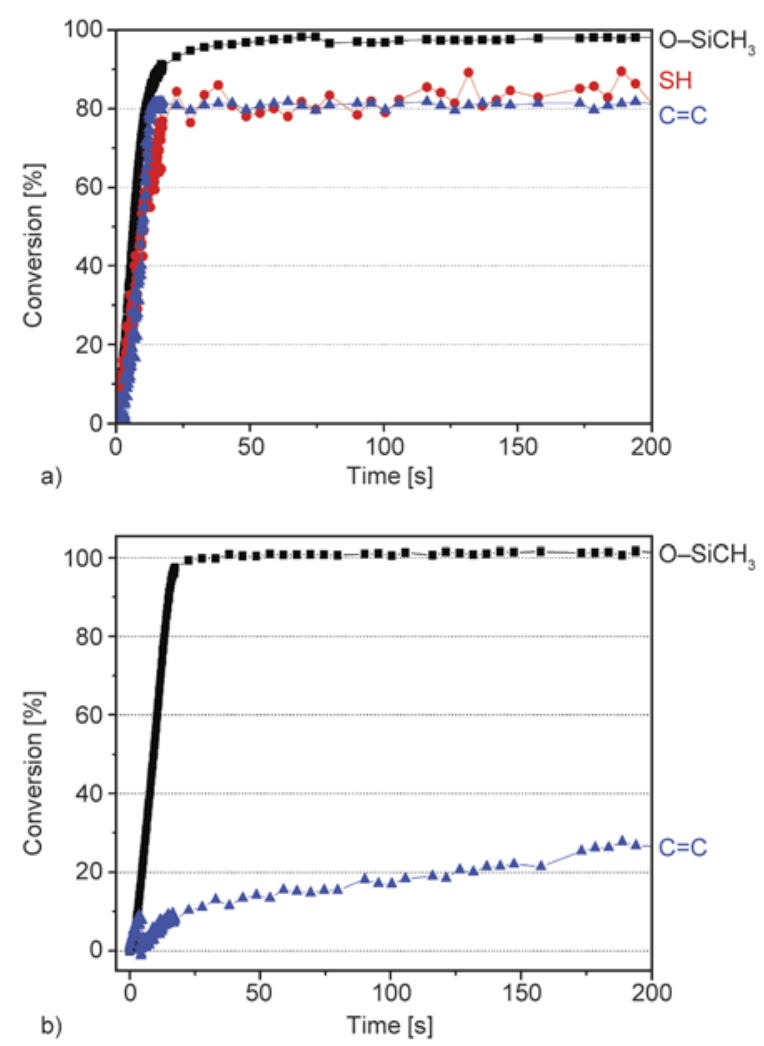

Figure 4. Temporal conversion in ene, thiol and methoxysilyl groups during the UV irradiation of $\mathbf{E}_{\mathbf{2}}$-T with a 1:1 thiol/ene stoichiometry (a). For comparison, kinetics of $\mathbf{E}_{\mathbf{2}}$ homopolymerization/sol-gel reaction is given (b). cals can be oxidized by $\mathrm{Ph}_{2} \mathrm{I}^{+}$. However, consumption of onium salt by this mechanism does not seem to affect the sol-gel progress.

Evidence of sol-gel condensation in the two nanocomposite thiol-ene films was indeed provided by ${ }^{29} \mathrm{Si}$ solid-state NMR. Figure 5 displays the quantitative ${ }^{29} \mathrm{Si}$ SPE-MAS NMR spectra of the two $\mathbf{E}_{\mathbf{1}}-\mathbf{T}$ and $\mathbf{E}_{\mathbf{2}} \mathbf{- T}$ nanocomposite films. In both systems, the broad resonances at $-48,-57$ and $-69 \mathrm{ppm}$ were straightforwardly assigned to $(\mathrm{XO})_{2}(\mathrm{R}) \mathrm{Si}(\mathrm{OSi})\left(\mathrm{T}^{1}\right)$, $(\mathrm{XO})(\mathrm{R}) \mathrm{Si}(\mathrm{OSi})_{2}\left(\mathrm{~T}^{2}\right)$ and $(\mathrm{R}) \mathrm{Si}(\mathrm{OSi})_{3}\left(\mathrm{~T}^{3}\right)$ where $\mathrm{R}$ is the organic component, and $\mathrm{X}$ is $\mathrm{CH}_{3}$ or $\mathrm{H}$ (after hydrolysis). The proportion of the different $\mathrm{T}^{\mathrm{n}}$ is provided in the same figure. A comparable and moderate condensation degree of $81 \%$ was estimated for the two thiol-ene hybrid films, supporting the efficiency of the photoinduced sol-gel reaction despite a high thiol-ene coupling yield. We note that similar levels were achieved with the two hybrid analogues obtained from the single ene organosilane precursors: $\mathbf{E}_{\mathbf{1}}(\mathbf{7 8 \%})$ and $\mathbf{E}_{\mathbf{2}}(80 \%)$, although much lower ene conversion was reported in this case $(<30 \%)$. Additionally, the formation of thioether bonds was investigated for the $\mathbf{E}_{\mathbf{1}} \mathbf{- T}$ pair by ${ }^{13} \mathrm{C}$ SPE-MAS NMR (data not given). The -C-S-C- connectivity was reflected by the almost disappearance of the two unsaturated carbons (120-130 ppm) from $\mathbf{E}_{\mathbf{1}}$ and the appearance of an intense resonance at $35 \mathrm{ppm}$ assigned to the two $\alpha$-carbons attached to sulfur, with a chemical shift significantly different from $\alpha$-carbon in thiols ( $25 \mathrm{ppm}$ for $-\mathbf{C}-\mathrm{SH}$ in precursor T).

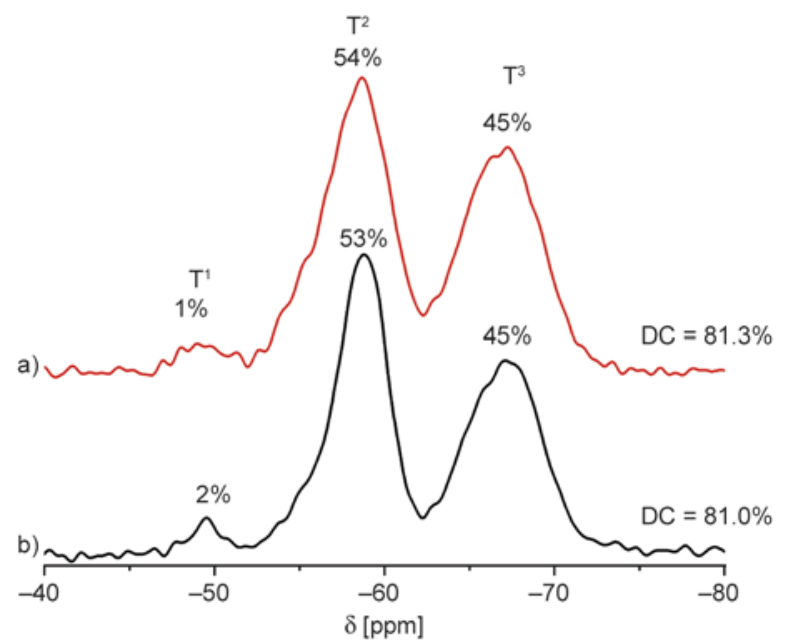

Figure 5. Solid-state ${ }^{29} \mathrm{Si}$ SPE MAS NMR spectra of UV crosslinked $\mathbf{E}_{\mathbf{1}}-\mathbf{T}$ (a) and $\mathbf{E}_{\mathbf{2}}$-T (b) thiol-ene hybrid films 


\subsection{Mechanical characterization}

There is a vast array of work on the mechanical properties of thiol-ene networks [28-30]. However, the majority is focused on homogeneity in the network crosslink density reflected by a narrowing of the loss tangent peak measured via dynamic mechanical analysis [31]. In our case, the surface mechanical properties of the cured hybrid films have been emphasized by means of scratch tests. For the preparation of the specimens, larger surfaces $(20 \times 20 \mathrm{~mm})$ were needed, and films were synthesized under a UV-conveyor (see experimental section). However, we checked that similar final conversions were obtained under these irradiation conditions. In order to evaluate the effect of thiol-ene coupling on film mechanical properties, $\mathbf{E}_{\mathbf{1}} \mathbf{- T}$ behavior was compared with $\mathbf{E}_{\mathbf{1}}$ film, both having comparable inorganic content $(27 \pm 2 \mathrm{wt} \%$.) and level of condensation $(81 \pm 2 \mathrm{~mol} \%)$.

As described in a separate publication [27], the 'MicroVisioScratch' set-up comprises a diamond tip draining across the film under increasing stepwise load, and coupled with in situ optical microscope observation to study the mechanical behavior and damage mechanism which appears during scratching. Figure 6 displays a set of in situ photo- graphs of the grooving tip taken during the scratch tests performed on these three films. The most relevant pictures are provided as well as the corresponding average contact pressure (i.e. the normal force contact surface ratio). For the thiol-ene hybrid film $\mathbf{E}_{\mathbf{1}} \mathbf{- T}$, three canonical mechanical domains can be observed. Below a threshold pressure of $2058 \mathrm{MPa}$, the elastic regime is maintained in which the film undergoes only a reversible deformation, and there is consequently neither residual scratching track nor damage after the passage of the tip. In this domain, the lack of plasticity results in a contact area forming an undistorted disk matching exactly the spherical shape of the tip (Image 1). Beyond $2058 \mathrm{MPa}$, appear consecutively the two usual forms of cracking: first blistering (Image 2) with a fracture mechanism restricted to the substrate/film interface. Above $2823 \mathrm{MPa}$, flaking occurs (Image 3) which reflects cohesive cracking within the film. By comparison, the film derived from single precursor $\mathbf{E}_{\mathbf{1}}$ reveals no elastic domain but a very narrow plastic region (Image $\mathbf{1}^{\prime}$ ). In this plastic domain, the contact area becomes dissymmetric showing an apparent front pad and the second distinctive point is a partial recovery of the groove left on the surface (Image 2'). For pressure greater than $115 \mathrm{MPa}$, flak-

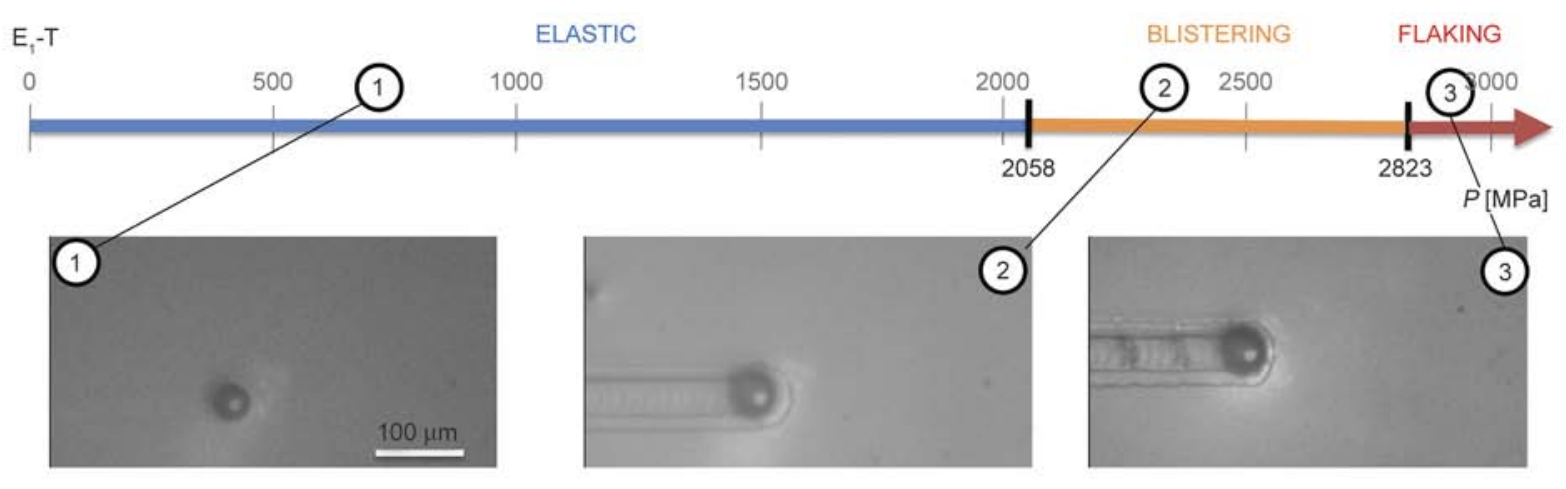

$E_{1}$

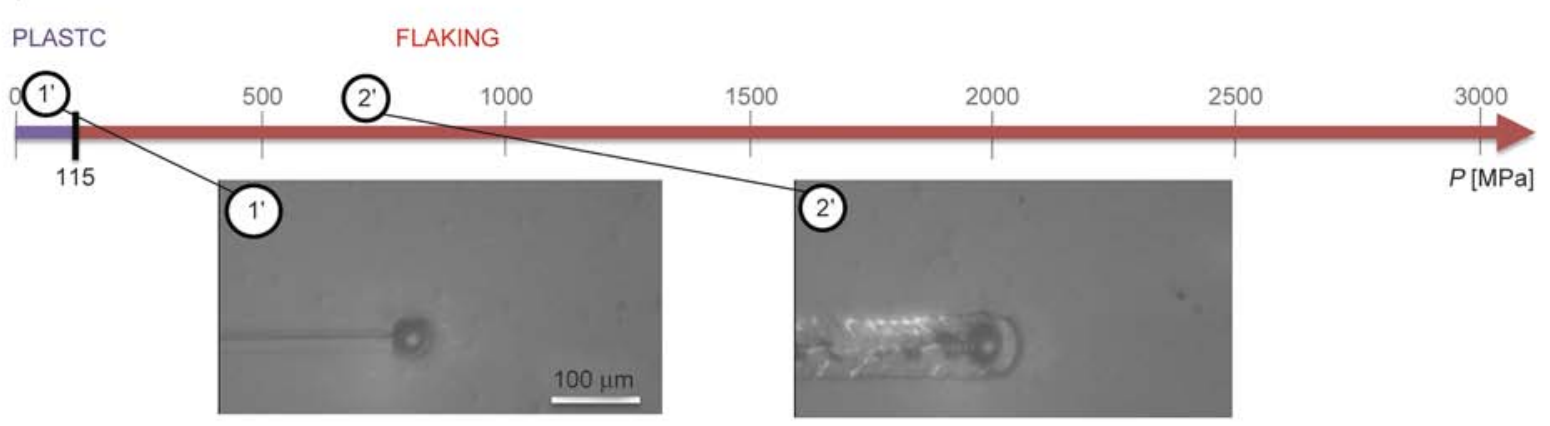

Figure 6. Difference of scratch resistance between thiol-ene coupled hybrid films $\left(\mathbf{E}_{\mathbf{1}}-\mathbf{T}\right)$ and homopolymer hybrid films $\left(\mathbf{E}_{1}\right)$ as a function of the average contact pressure $(P)$. The different regimes (elastic, blistering and cracking) are reported. 
ing takes place (Image 2'). It is well-established that this critical pressure or load, generating the first damage in a scratch test, is representative of the behavior of a coating. As conclusion, this first methacrylate system $\left(\mathbf{E}_{\mathbf{1}}-\mathbf{T} v s \mathbf{E}_{\mathbf{1}}\right)$ shows thiol-ene as a very efficient approach for the formation of crosslinked materials with enhanced mechanical properties. In glassy silsesquioxane networks, the residual stress may be important and modes of dissipation are frozen out and unable to accommodate deformation without the formation of cracks. In the $\mathbf{E}_{\mathbf{1}} \mathbf{- T}$ thiolene system, we hypothesize that the introduction of flexible thioether bridges provides more molecular mobility and may balance the rigidity of the glassy siloxane network. While flexible connective organic groups are able to extent elasticity domain and retard cracking, rigid carbon-carbon single bonds and more crosslinked of polymethacrylate main chain $\left(\mathbf{E}_{1}\right)$ do not give the ability for polymer segments to move and dissipate energy deformation, thus causing mechanical failure at low loading.

The further advantage of the thiol-ene hybrid can be illustrated by varying the proportion between the two precursors: $\mathbf{E}_{\mathbf{1}}$ and $\mathbf{T}$ as an easy means to modulate the proportion between ene homopolymerization and ene coupling. Where appropriate, Figure 7 shows the extent of the elastic, blistering and flaking domains as a function of the average contact pressure for six different hybrid films produced with different ene molar fractions: $0 \%(\mathbf{T}), 20,40$,

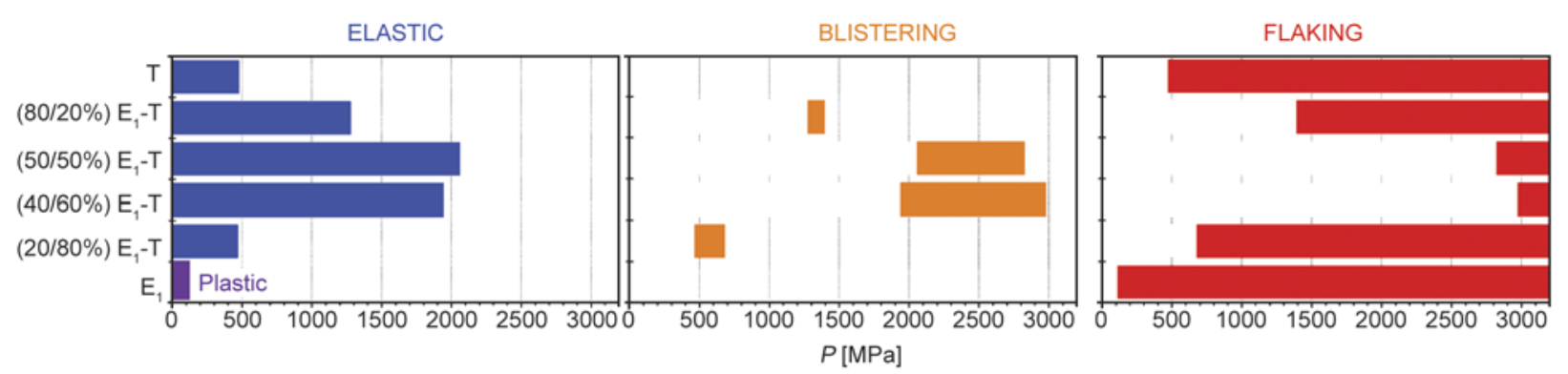

Figure 7. Effect of thiol and ene monomer proportion on elasticity, blistering and flaking of $\mathbf{E}_{\mathbf{1}}$-T hybrid films during scratch tests. Data are plotted as a function of the average contact pressure in $\mathrm{MPa} .(x / y[\%]) \mathbf{E}_{\mathbf{1}}-\mathbf{T}$ is associated with a hybrid film including $x[\%]$ of ene and $y[\%]$ of thiol groups.

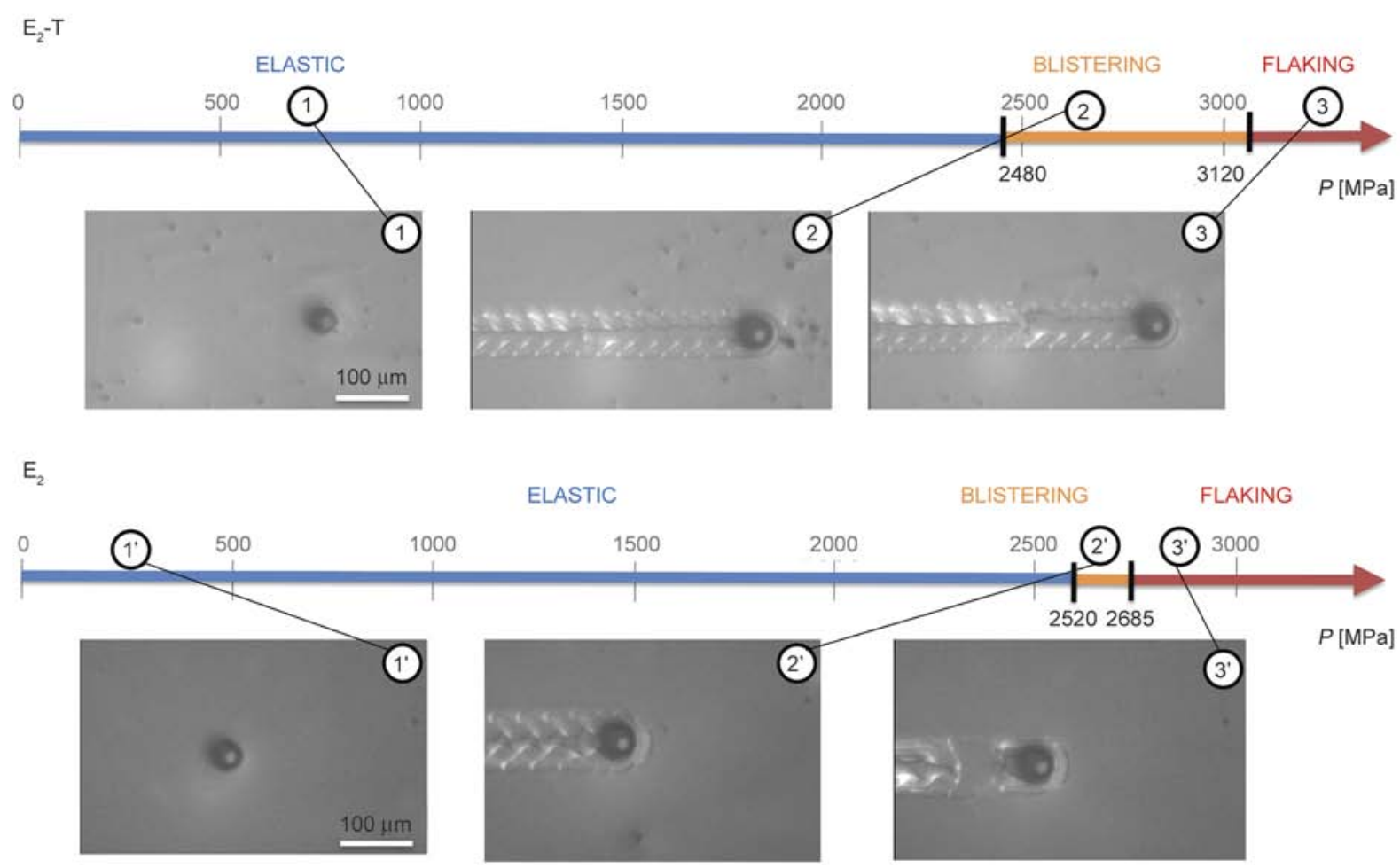

Figure 8. Difference of scratch behavior between thiol-ene hybrid films $\mathbf{E}_{\mathbf{2}} \mathbf{- T}$ and its homologue based on radical chaingrowth polymerization $\mathbf{E}_{\mathbf{2}}$ depending on average contact pressure $(P)$ 


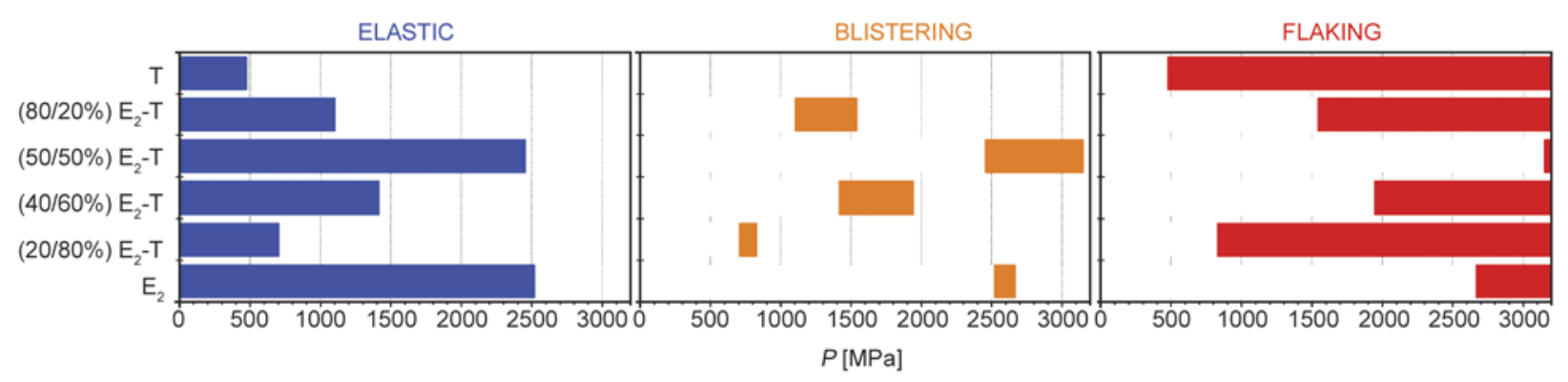

Figure 9. Extent of elastic, blistering and flaking domains in $\mathbf{E}_{\mathbf{2}}$-T hybrid films as a function of the thiol/ene molar proportions $(x / y[\mathrm{~mol} \%])$

$50 \%\left(\mathbf{E}_{\mathbf{1}}-\mathbf{T}_{\mathbf{1}}\right), 80$ and $100 \%\left(\mathbf{E}_{\mathbf{1}}\right)$. There is clearly a progressive extension of the elastic domain, and better resistance to cracking as the ratio of thiol and ene functions approximates $1\left(\mathbf{E}_{\mathbf{1}}-\mathbf{T}_{\mathbf{1}}\right)$. The utility of forming thioether bridges within a silsesquioxane is thus demonstrated as a way to create a uniform, low-stress and flexible crosslinked hybrid structure. Moreover, there are clear indications that mechanical properties can be easily modulated. We see the versatility of the approach enabling to tune mechanical properties of polysulfide-crosslinked materials with varying the ratio of the two precursors.

Nevertheless, generalization of the findings of the $\mathbf{E}_{1}$ - $\mathbf{T}$ couple to other thiol-ene couples seems challenging. For example, the $\mathbf{E}_{\mathbf{2}} \mathbf{-} \mathbf{T}$ thiol-ene film reveals somewhat similar elasticity, and cracking resistance compared to its homopolymerized homologue $\mathbf{E}_{\mathbf{2}}$ (Figure 8). In this second system, we clearly see that the added value of a flexible thioether linkage is less obvious, because the polyacrylate formed in $\mathbf{E}_{\mathbf{2}}$ has a much lower glass transition temperature than that of the polymethacrylate of $\mathbf{E}_{\mathbf{1}}$. This difference is attributed to stiffening of main chain of the polymer by the $\alpha$-methyl substituent of the polymethacrylate. However, Figures 9 illustrates again how mechanical properties can be tailored by changing the proportion of the two precursors, with clearly the best performances achieved with the thiol-ene hybrid film having 1:1 thiol ene molar ratio.

\section{Conclusions}

An orthogonal coupling strategy was developed by combining thiol-ene and sol-gel photoinduced reactions, which was subsequently applied to the one-pot synthesis of uniform and low stress hybrid films. Starting from two bifunctional meth(acrylate) and thiol trimethoxysilane precursors, thiol-ene has proven to be an efficient ligation tool to bind the two resultant silsesquioxane networks together through a single coupled thioether product. In this approach the sol-gel process enables the formation of a rigid oxo-polymer siloxane network with high crosslink density, while the thiol-ene coupling imparts flexibility, thus improving elasticity and resistance to cracking of the final material. The interest of using (meth)acrylate organosilane precursors is that thiolene and conventional chain-growth polymerization can be combined with the ability to tailor mechanical response.

\section{References}

[1] Hoyle C. E., Lowe A. B., Bowman C. N.: Thiol-click chemistry: A multifaceted toolbox for small molecule and polymer synthesis. Chemical Society Reviews, 39, 1355-1387 (2010). DOI: $10.1039 / \mathrm{b} 901979 \mathrm{k}$

[2] Dénès F., Pichowicz M., Povie G., Renaud P.: Thiyl radicals in organic synthesis. Chemical Review, 114, $2587-$ 2693 (2014).

DOI: $10.1021 / \mathrm{cr} 400441 \mathrm{~m}$

[3] Wong C-H., Zimmerman S. C.: Orthogonality in organic, polymer, and supramolecular chemistry: From Merrifield to click chemistry. Chemical Communications, 49, 1679-1695 (2013). DOI: $10.1039 / \mathrm{c} 2 \mathrm{cc} 37316 \mathrm{e}$

[4] Hoyle C. E., Bowman C. N.: Thiol-ene click chemistry. Angewandte Chemie International Edition, 49, 15401573 (2010).

DOI: 10.1002 /anie.200903924

[5] Jasinski F., Lobry E., Tarablsi B., Chemtob A., CroutxéBarghorn C., Le Nouen D., Criqui A.: Light-mediated thiol-ene polymerization in miniemulsion: A fast route to semicrystalline polysulfide nanoparticles. ACS Macro Letters, 3, 958-962 (2014).

DOI: $10.1021 / \mathrm{mz} 500458 \mathrm{~s}$

[6] DeForest C. A., Polizzotti B. D., Anseth K. S.: Sequential click reactions for synthesizing and patterning threedimensional cell microenvironments. Nature Materials, 8, 659-664 (2009).

DOI: $10.1038 /$ nmat 2473 
[7] Stenzel M. H.: Bioconjugation using thiols: Old chemistry rediscovered to connect polymers with nature's building blocks. ACS Macro Letters, 2, 14-18 (2013). DOI: $10.1021 / \mathrm{mz} 3005814$

[8] Brinker C. J., Scherer G. W.: Sol-gel science. Academic Press, San Diego (1990).

[9] Sangermano M., Colucci G., Fragale M., Rizza G.: Hybrid organic-inorganic coatings based on thiol-ene systems. Reactive and Functional Polymers, 69, 719723 (2009).

DOI: $10.1016 /$ j.reactfunctpolym.2009.05.008

[10] Moszner N., Schöb W., Rheinberger V.: Synthesis, characterization and thiol-ene polymerization of hydrolyzed/ condensed norbornenyl silic acid ester. Polymer Bulletin, 37, 289-295 (1996).

DOI: $10.1007 / \mathrm{bf} 00318060$

[11] Kim J-S., Yang S., Park H., Bae B-S.: Photo-curable siloxane hybrid material fabricated by a thiol-ene reaction of sol-gel synthesized oligosiloxanes. Chemical Communications, 47, 6051-6053 (2011).

DOI: $10.1039 / \mathrm{c} 1 \mathrm{cc} 11297 \mathrm{j}$

[12] Schreck K. M., Leung D., Bowman C. N.: Hybrid organic/inorganic thiol-ene-based photopolymerized networks. Macromolecules, 44, 7520-7529 (2011). DOI: $10.1021 / \mathrm{ma} 201695 \mathrm{x}$

[13] Cole M. A., Bowman C. N.: Synthesis and characterization of thiol-ene functionalized siloxanes and evaluation of their crosslinked network properties. Journal of Polymer Science Part A: Polymer Chemistry, 50, 43254333 (2012).

DOI: $10.1002 /$ pola.26245

[14] Kim J-S., Lee S., Hwang Y. H., Kim Y., Yoo S., Bae BS.: Photo-curable sol-gel hybrid film as a dielectric layer by a thiol-ene reaction in air or $\mathrm{N}_{2}$ for organic thin film transistors. Electrochemical and Solid-State Letters, 15, G13-G15 (2012).

DOI: $10.1149 / 2.021205 \mathrm{esl}$

[15] Sparks B. J., Kuchera T. J., Jungman M. J., Richardson A. D., Savin D. A., Hait S., Lichtenhan J., Striegel M. F., Patton D. L.: Cyclic tetravinylsiloxanetetraols as hybrid inorganic-organic thiol-ene networks. Journal of Materials Chemistry, 22, 3817-3824 (2012).

DOI: $10.1039 / \mathrm{c} 2 \mathrm{jm} 15484 \mathrm{f}$

[16] Igarashi Y., Kajihara K., Kanamura K.: Thiol-containing polysilsesquioxane liquid and photocurable sulfurcontaining transparent organic-inorganic hybrid monoliths obtained via cosolvent-free hydrolytic polycondensation. Bulletin of the Chemical Society of Japan, 86, 880-883 (2013).

DOI: $10.1246 /$ bcsj.20130053

[17] Peng S., Zeng Z., Zhao W., Li H., Chen J., Han J., Wu X.: Novel functional hybrid silica sol-gel coating for copper protection via in situ thiol-ene click reaction. RSC Advances, 4, 15776-15781 (2014).

DOI: $10.1039 / \mathrm{c} 4 \mathrm{ra} 00142 \mathrm{~g}$
[18] Clark T. S., Hoyle C. E., Nazarenko S.: Kinetics analysis and physical properties of photocured silicate-based thiol-ene nanocomposites: The effects of vinyl POSS ene on the polymerization kinetics and physical properties of thiol-triallyl ether networks. Journal of Coatings Technology and Research, 5, 345-351 (2008). DOI: $10.1007 / \mathrm{s} 11998-008-9092-\mathrm{x}$

[19] Li L., Liang R., Li Y., Liu H., Feng S.: Hybrid thiol-ene network nanocomposites based on multi(meth)acrylate POSS. Journal of Colloid and Interface Science, 406, 30-36 (2013).

DOI: $10.1016 /$ j.jcis.2013.05.044

[20] Sangermano M., Gross S., Priola A., Rizza G., Sada C.: Thiol-ene hybrid organic/inorganic nanostructured coatings based on thiol-functionalized zirconium oxoclusters. Macromolecular Chemistry and Physics, 208, 2560-2568 (2007).

DOI: $10.1002 / \mathrm{macp} .200700323$

[21] Esposito Corcione C., Striani R., Frigione M.: Sunlight curable hybrid organic-inorganic methacrylic-based coatings: Analysis of the cure mechanism and functional properties. Polymers for Advanced Technologies, 26, 167-175 (2015).

DOI: $10.1002 /$ pat.3445

[22] Lowe A. B.: Thiol-ene 'click' reactions and recent applications in polymer and materials synthesis: A first update. Polymer Chemistry, 5, 4820-4870 (2014). DOI: $10.1039 / \mathrm{c} 4$ py00339j

[23] Bhagat S. D., Chatterjee J., Chen B., Stiegman A. E.: High refractive index polymers based on thiol-ene cross-linking using polarizable inorganic/organic monomers. Macromolecules, 45, 1174-1181 (2012). DOI: $10.1021 / \mathrm{ma} 202467 \mathrm{a}$

[24] Chemtob A., Versace D-L., Belon C., Croutxé-Barghorn C., Rigolet S.: Concomitant organic-inorganic UVcuring catalyzed by photoacids. Macromolecules, 41, 7390-7398 (2008).

DOI: $10.1021 / \mathrm{ma} 801017 \mathrm{k}$

[25] De Paz H., Chemtob A., Croutxé-Barghorn C., Le Nouen S., Rigolet S.: Insights into photoinduced solgel polymerization: An in situ infrared spectroscopy study. Journal of Physical Chemistry B, 116, 52605268 (2012). DOI: $10.1021 / \mathrm{jp} 212386 \mathrm{e}$

[26] Massiot D.: Sensitivity and lineshape improvements of MQ-MAS by rotor-synchronized data acquisition. Journal of Magnetic Resonance, Series A, 122, 240-244 (1996).

DOI: $10.1006 /$ jmra.1996.0202

[27] Le Houérou V., Gauthier C., Schirrer R.: Mechanical analysis of the blistering of a thin film deposited on a glassy polymer. Tribology International, 43, 129-135 (2010).

DOI: $10.1016 / \mathrm{j}$. triboint.2009.05.011 
[28] Kasprzak S. E., Martin B., Raj T., Gall K.: Synthesis and thermomechanical behavior of (qua)ternary thiolene(/acrylate) copolymers. Polymer, 50, 5549-5558 (2009).

DOI: 10.1016/j.polymer.2009.09.044

[29] McNair O. D., Janisse A. P., Krzeminski D. E., Brent D. E., Gould T. E., Rawlins J. W., Savin D. A.: Impact properties of thiol-ene networks. ACS Applied Materials and Interfaces, 5, 11004-11013 (2013).

DOI: $10.1021 / \mathrm{am} 403238 \mathrm{~g}$
[30] An S. Y., Lee D. G., Hwang J. W., Kim K. N., Nam J. H., Jung H. W., Noh S. M., Oh J. K.: Photo-induced thiol-ene polysulfide-crosslinked materials with tunable thermal and mechanical properties. Journal of Polymer Science Part A: Polymer Chemistry, 52, 30603068 (2014).

DOI: $10.1002 /$ pola.27353

[31] Senyurt A. F., Wei H., Phillips B., Cole M., Nazarenko S., Hoyle C. E., Piland S. G., Gould T. E.: Physical and mechanical properties of photopolymerized thiol-ene/ acrylates. Macromolecules, 39, 6315-6317 (2006). DOI: $10.1021 / \mathrm{ma} 060507 \mathrm{f}$ 